\title{
Indigenous Australians and Impaired Decision-Making Capacity
}

Natalie Clements, Jayne Clapton and Lesley Chenoweth

\section{Abstract}

This article explores issues associated with impaired decision-making capacity for Indigenous Australians. There is very little published on the subject of impaired decision-making capacity in Australia, particularly in relation to Indigenous people. To gain some insight into this subject, this article looks at some indicators of impaired decision-making capacity for Indigenous Australians such as rates of intellectual disability and mental illness. The Australian state-based Guardianship and Administration system -the legislative framework designed to provide for decision-making for people with impaired capacity_-is briefly described before looking at the cultural relevance of this Western system and its constructs for Indigenous Australians. Future investigation should be directed at exploring existing and alternative strategies to support Indigenous Australians with impaired capacity and their carers.

Keywords: Indigenous; mental capacity; substitute decision-making. 
This article explores indicators of the extent to which Indigenous Australians experience impaired decision-making capacity (impaired capacity), which is a complex legal concept embedded in a statutory framework, the Guardianship and Administration system. This system is designed to provide guidance to assist people with impaired capacity for the administration of particular decisions, usually around personal, health and financial issues. The Guardianship and Administration legal framework in Australia provides for a range of substitute decision-making and supported decision-making mechanisms for people with impaired capacity. Little research has been conducted on the legal notion of impaired capacity within Australia, particularly on indicators of impaired capacity for Indigenous Australians, or on the cross-cultural relevance of this concept. Whether impaired capacity as currently understood in a Western framework is relevant for Indigenous Australians is discussed.

\section{Decision-making Capacity}

The notion of decision-making capacity has evolved as a legal construct in liberal democracies as the means for specifying which people (usually those with an intellectual disability or mental illness) may require being taken care of by others (Silberfeld \& Checkland 1999). Legal definitions of mental capacity have moved from viewing mental capacity as a global, all-embracing condition to a more specific condition restricted to particular realms of decision-making, usually around personal, health and financial types of decisions. Although defined by legislation, capacity is usually assessed by a psychologist or psychiatrist who tests a person's cognitive abilities. Moberg and Kniele (2006) note that there is much debate among practitioners as to which combination of cognitive abilities comprises decision-making capacity, and therefore there is much variability in the methods and measures used to evaluate decision-making capacity in the clinical context.

Decision-making capacity is now, in general, defined in terms of functional abilities to understand information relevant to a particular decision, and to appreciate the consequences of the presenting options. As an example of a legislative definition of capacity, Schedule 4 of the Queensland Guardianship and Administration Act 2000 states that capacity, for a person for a matter, means the person is capable of-

(a) understanding the nature and effect of decisions about the matter; and

(b) freely and voluntarily making decisions about the matter; and

(c) communicating the decisions in some way.

A person with impaired capacity may have one or more of the following: an intellectual disability; an acquired brain injury (ABI); a history of drug and alcohol abuse which has impaired neurological functioning; dementia; a mental illness that impacts on the ability to make decisions; emotional and/or physical trauma (which may be past or present); or a developmental disorder such as Autism Spectrum Disorder or Asperger's Disorder. 


\section{Indicators of Impaired Capacity}

Data on the incidence of intellectual disability, mental illness, dementia, Foetal Alcohol Syndrome, acquired brain injury, petrol sniffing and other substance abuse problems may provide an indication of the levels of impaired capacity among Indigenous Australians. Unfortunately, there is a relative lack of robust data on these issues. For example, a recent federal inquiry into petrol sniffing states that the absence of statistical data and full and accurate records of petrol sniffing in Indigenous communities makes it difficult to determine the full impact of petrol sniffing (Senate Community Affairs References Committee 2006). Some indicators, however, are provided below.

A study on intellectual disability among the Indigenous population of Western Australia found that although comprising 3.5 percent of the population, Indigenous Australians represented 7.4 percent of all people registered for intellectual disability services (Glasson et al. 2005). In the 2002 National Aboriginal and Torres Strait Islander Social Survey conducted by the Australian Bureau of Statistics (ABS), intellectual disability was estimated to affect 19,600 Indigenous people nationally. Indigenous people with intellectual disability experienced core activity limitations and restrictions more commonly, and to a greater extent, than Indigenous people with other disabilities or long-term health conditions (Australian Institute of Health and Welfare 2007).

Additionally, using data from the 1993 ABS Survey of Disability, Ageing and Carers, the Australian Institute of Health and Welfare has estimated that 1-1.9 percent of the Australian Indigenous population has an ABI-related disability (Australian Institute of Health and Welfare 1999), and a recent study in the Kimberley region of Western Australia found a higher prevalence of dementia among Indigenous people than in the general population (Smith et al. 2007). Only recently have data been published on Foetal Alcohol Syndrome in Australia, and they have highlighted the over-representation of Indigenous children with Foetal Alcohol Syndrome (Elliott et al. 2008). Findings from a five-year study of cannabis users in Arnhem Land, Northern Territory, were that ongoing heavy cannabis use is commonplace in the already vulnerable Indigenous communities (Lee et al. 2009).

Drawing on the 2004-05 National Aboriginal and Torres Strait Islander Health Survey and the National Hospital Morbidity Database relating to the social and emotional wellbeing of Indigenous Australians, the Australian Institute of Health and Welfare (2009) reports the following key findings:

- 27 per cent of Indigenous adults reported high or very high levels of psychological distress;

- Indigenous Australians were twice as likely to report high or very high levels of psychological distress as non-Indigenous Australians, and

- In 2005-2007 mental and behavioural disorders due to psychoactive substance use were the most common type of mental health-related conditions for which Indigenous Australians were hospitalised (38 per cent of hospitalisations). 
From these reported statistics, it seems fair to assume that there would be many Australian Indigenous people whose disability, disorder or condition would fit the legal definition of impaired capacity. The factors contributing to reported greater levels of disability, mental illness, dementia and acquired brain injury are likely to be linked to the extreme marginalisation and social disadvantage experienced by many Indigenous people since European settlement (Tipper \& Dovey 1991; Simpson \& Sotiri 2004; Vicary \& Bishop 2005). Important factors that have affected the social and emotional wellbeing of Indigenous Australians may include the introduction of custodial care (Australian Institute of Health and Welfare 2009) and the over-representation of Australian Indigenous children in the child welfare system (Tilbury 2009).

In Australian states there are mixtures of three legislative frameworks for covering any loss of a citizen's presumed decision-making capacity (Carney 2004). The public planning option, which may involve the appointment of a statutory agency as a guardian of last resort; the private planning option, which involves designating a private citizen as an enduring power of attorney (for financial affairs), an enduring guardian (for personal affairs) or an enduring health power (health affairs); and the person responsible model, where lack of decision-making capacity is handled through a presumed appointment of family/ friends.

The state-based Australian Guardianship and Administration scheme, designed to protect people with impaired capacity primarily through the public planning option, is relatively new, having been developed over the past twenty years (House of Representatives Standing Committee on Legal and Constitutional Affairs 2007). The Guardianship and Administration legal framework provides for a range of substitute decision-making and supported decision-making mechanisms for people with impaired capacity. Guardianship legislation applies to certain types of decisions, usually relating to personal, health or financial issues.

With supported decision-making, the presumption of capacity is always in favour of the person with a disability who will be affected by the decision, and support should enable the individual to exercise his/her legal capacity (United Nations 2007). Substitute decision-making, which may rely on advance directives and guardians who have court-authorised power to make decisions on behalf of the individual, does not have to demonstrate that those decisions are in the individual's best interests or according to his/her wishes.

\section{Cross-cultural Relevance of 'Impaired Capacity'}

The Human Rights and Equal Opportunity Commission's (2005) review of Indigenous young people with cognitive disabilities within the Australian juvenile justice system highlighted the issues of the cultural inappropriateness of most assessment tools. Cognitive assessments usually use language, stimuli or normative data with a cultural bias towards non-Indigenous people, and therefore may produce misdiagnosis among those from other cultures. Performance deficits on tasks that suggest brain dysfunction may actually arise 
from cultural determinants for Indigenous people such as socio-economic status, language or expectations of the assessment experience (Cairney \& Maruff 2007).

Cattarinich et al. (2001) argue that both the process by which capacity assessments are conducted and the content of the assessment instruments are problematic cross-culturally. Cultural considerations for capacity assessments of Indigenous Australians include familiarity with ethnic protocols such as communication styles and taboos. As an example, due to the Indigenous preference for indirect communications (e.g. using metaphors and stories), a non-Indigenous professional may not realise when a patient has in fact answered a question, nor what the response means (Hepburn \& Reed 1995). Only recently have cognitive assessment tools for use specifically with Indigenous Australians been developed, such as CogState (Human Rights and Equal Opportunity Commission 2005), Biala-II (Shaddock et al. 2000), and the Kimberley Indigenous Cognitive Assessment (KICA) (Lo Guidice et al. 2006; Smith et al. 2007)

There have been a few investigations of Indigenous definitions and understandings of disability and/or mental illness in Australia. For example, Senior (2000) has undertaken fieldwork with Indigenous communities in the Northern Territory to understand Indigenous perspectives of disability and/or mental illness. Ariotti (1999) has examined the social construction of disability among the Anangu people of the cross border region of Western Australia, South Australia and the Northern Territory. However, no studies have been specific to Indigenous understandings of impaired capacity.

\section{Indigenous Interaction with the Australian Guardianship and Administration System}

With the exception of the state of Western Australia, no Australian states or territories have publicly available figures on the number of Indigenous persons that have been involved in the Guardianship and Administration scheme. Western Australia and Queensland are the only states to have provisions in their Guardianship and Administration legislation mandating the consideration of a person's cultural environment when determining the best interests of a represented person. Additionally, there is no reference to the Australian Guardianship scheme or to Indigenous Australians with impaired capacity in the Australian government's key strategy to address Indigenous disadvantage and social exclusion, the National Indigenous Reform Agreement (Council of Australian Governments 2008).

In 2001, the first Australian investigation into the relevance and appropriateness of the Guardianship and Administration system to Western Australian Indigenous people with a decision-making disability was conducted (Other-Gee et al. 2001). The research consisted of a survey of Indigenous agencies and service providers as well as consultations with Indigenous people and their families in rural and remote parts of the state. The research found evidence of growing levels of decision-making disability resulting from the combined effects 
of severe disadvantage associated with substance abuse, early ageing, psychiatric disability, brain damage and traumatic life events such as motor vehicle accidents (Other-Gee et al. 2001).

Other-Gee et al. (2001) also found that there was an under-representation of Indigenous people in the Western Australian Guardianship and Administration system. They suggested the following reasons for this finding:

- a lack of awareness of the system and its function by Indigenous people;

- a lack of cultural relevance of the system; and

- the inherent difficulties of a statutory system in the Indigenous context, when many past and present government policies have had a negative effect on Indigenous people and communities.

Cunneen (2005) provides additional support for this last point, noting that historically the powers under various Aborigines' Protection Acts had instituted legal regimes that provided for the total control of Indigenous activities, and which presumed that Indigenous people had impaired capacity in general. This may, in part, explain any reluctance on the part of Indigenous Australians to utilise the Guardianship and Administration system.

Other-Gee and colleague's (2001) findings are supported by Setterlund et al. (1999) who, in a study of older people's knowledge of the substitute decision-making process and experiences of abuse, suggest that there are specific cultural factors that reduce access to, and the response of, the Guardianship system for Indigenous people. These include:

- cultural beliefs in the inappropriateness of taking family issues outside of the family;

- a belief that appointing an individual as a substitute decision-maker for something that is viewed as a communal responsibility is inappropriate;

- language barriers, and

- lack of information and awareness about the law and civil justice remedies.

Additionally, the Law Reform Commission of Western Australia (the Commission) (2006) recently reported that concerns have been raised about the application and accessibility of the Guardianship and Administration system to Indigenous people in that state, including the system's interaction with Indigenous customary laws and cultural beliefs. The Commission found that issues for the Western Australian system to address are:

- concern from agency staff about intervening in Indigenous family systems given the historical context of the effect of government policies on Indigenous people;

- understanding cultural norms, for example, communal sharing and reciprocal obligation;

- knowing who to talk to within the kinship system; and 
- the system dealing with failings of other systems of care and/or services, for example, when a person is passed between the homeless service system, the criminal justice system, and the mental health system, without getting their needs addressed.

\section{Alternative Approaches for Indigenous Australians to Engage with the Guardianship and Administration Scheme}

Both of the investigations in Western Australia (Other-Gee et al. 2001; Law Reform Commission of Western Australia 2006) concluded that there is, to some extent, a fundamental incompatibility between the values, intentions and operations of the Guardianship system and the aspirations, experiences and social and cultural realities of Indigenous people. In particular, there is a need for specific protocols to be established to ensure that cultural aspects of competency are considered in assessment of capacity. Other-Gee et al. (2001) also suggest that there may be ways in which the Guardianship and Administration system could more effectively support and strengthen the informal supported and substitute decision-making arrangements already in place for some Indigenous people, by enabling local service providers to assist Indigenous families to meet the needs of their family members.

An example of an informal arrangement for substitute decision-making in an Indigenous community is available from a recent hearing in Western Australia (FS [2007] WASAT 202, p 18):

Mr S's family and his community have expressed a desire to assist $\mathrm{Mr} \mathrm{S}$ in making appropriate financial decisions by using the 'old way' which is a process of meetings between $\mathrm{Mr} \mathrm{S}$, his family and the relevant elder to discuss financial issues and to attempt to reach a consensus (with, however, $\mathrm{Mr} \mathrm{S}$ having the final say on how the money is used).

In 2004, Queensland Advocacy Inc consulted with representatives of Indigenous communities in Queensland and recommended that mechanisms be identified to protect and safeguard Indigenous people with impaired capacity who are in receipt of large compensation payments or funding. These mechanisms might include the creation of trusts and the development of resources for community legal services and community workers (Queensland Advocacy Inc 2004), and could be used to supplement the formal Guardianship and Administration system.

\section{Conclusion}

Although the data are somewhat limited, given the numbers of Indigenous people with indicators of impaired capacity, such as intellectual disability and Foetal Alcohol Syndrome, it is reasonable to assume there are some (and perhaps a significant number) of Indigenous Australians with impaired capacity. It is unclear whether the Guardianship and Administration system in Australia is much utilised by Indigenous people, but such information would provide another indicator of the incidence of impaired capacity among 
Indigenous Australians, and it would be useful if agencies collected data on this. Additionally, while there is a need for more research, early indications from this overview suggest that the Guardianship and Administration system may not be meeting Indigenous peoples' needs because of cultural barriers, such as the misfit between the legislation and Indigenous concepts such as cultural obligation.

There may be a need for a broader range of decision-making alternatives to be developed for Indigenous people. Some alternative approaches might be designed around involving the local Indigenous community and increasing support for informal supported and/or substitute decision-making arrangements, such as providing funding for community resources to aid in planning.

Given the likelihood there may be significant need, urgent research and attention are required focusing on:

- ways of improving data collection regarding indicators of impaired capacity for Indigenous Australians such as the incidence of substance misuse and related neurological impairment; as well as the numbers of Indigenous Australians accessing and utilising the Australian Guardianship and Administration system;

- the cultural relevance of the construct of impaired decision-making capacity;

- the cultural relevance of the existing Guardianship and Administration system;

- existing decision-making processes used by Indigenous Australians to assist those with impaired capacity around personal, health and financial decisions;

- possibilities for alternative arrangements that are locally developed with support provided by government;

- the level of awareness and understanding among Indigenous Australians of the legal framework for supported and substitute decision-making in Australian States and Territories; and

- analysis of any difficulties experienced by Indigenous Australians when engaging with the Guardianship and Administration system.

Considering the historical context in Australia where important life decisions for Indigenous Australians were made by the State, the Guardianship and Administration system may be seen by Indigenous Australians as a contemporary form of the previous "protectionist" legislative framework. Future investigation of the issues outlined in this paper will need to involve Indigenous Australians in order to design and deliver relevant and responsive services.

\section{Acknowledgements}

Office of the Public Advocate Queensland. 


\section{References}

Ariotti, L. (1999) 'Social construction of Anangu disability', Australian Journal of Rural Health, 7 (4), 216-22.

Australian Institute of Health and Welfare (1999) The Definition, Incidence and Prevalence of Acquired Brain Injury in Australia, Canberra, Australian Institute of Health and Welfare.

Australian Institute of Health and Welfare (2007) 'Intellectual disability in Australia's Aboriginal and Torres Strait Islander peoples', Journal of Intellectual and Developmental Disability, 32, 222-5.

Australian Institute of Health and Welfare (2009) Measuring the Social and Emotional Wellbeing of Aboriginal and Torres Strait Islander Peoples, Canberra, Australian Institute of Health and Welfare.

Cairney, S. \& Maruff, P. (2007) 'Computerised tests of brain function for use with Indigenous people’. In L.E. Dyson, M. Hendricks \& S. Grant [eds] Information Technology and Indigenous people, Hershey PA, IDEA Group Inc.

Carney, T. (2004) 'Aged capacity and substitute decision-making in Australian and Japan', Law Asia Journal, 2003/041-21.

Cattarinich, X., Gibson, N. \& Cave, A.J. (2001) 'Assessing mental capacity in Canadian Aboriginal seniors', Social Science \& Medicine, 53 (11), 1469-79.

Council of Australian Governments (2008) National Indigenous Reform Agreement (Closing the Gap), Canberra, Council of Australian Governments.

Cunneen, C. (2005) 'Colonialism and historical injustice', Social Semiotics, 15(1), 59-80.

Elliott, E., Payne, J.M., Morris, A., Hann, E. \& Bower, C.A. (2008) 'Foetal Alcohol Syndrome: A prospective national surveillance study', Archives of Disease in Childhood, 93, 732-7.

Glasson, E.J., Sullivan, S.G., Hussain, R. \& Bittles, A.H. (2005) 'An assessment of intellectual disability among Indigenous Australians', Journal of Intellectual Disability Research, 49, 626-34.

Hepburn, K., \& Reed, R. (1995) 'Ethical and clinical issues with Native-American elders: End of life decision making', Clinics in Geriatric Medicine, 11 (1), 97-111.

House of Representatives Standing Committee on Legal and Constitutional Affairs (2007) Older People and the Law, Canberra, Parliament of the Commonwealth of Australia.

Human Rights and Equal Opportunity Commission (2005) Indigenous young people with cognitive disabilities and Australian juvenile justice systems, Sydney, Human Rights and Equal Opportunity Commission. 
Law Reform Commission of Western Australia (2006) Aboriginal Customary Laws: The Interaction of Western Australian Law with Aboriginal Law and Culture, Perth, Law Reform Commission of Western Australia.

Lee, K.S., Conigrave, K.M., Clough, A.R., Dobbins, T.A., Jaragba, M.J., \& Patton, G.C. (2009) 'Five-year longitudinal study of cannabis users in three remote Aboriginal communities in Arnhem Land, Northern Territory, Australia', Drug and Alcohol Review, 28, 623-30.

Lo Guidice, D., Smith, K., Thomas, J., Lautenschlager, N.T., Almeida, O.P., Atkinson, D. \& Flicker, L. (2006) 'Kimberley Indigenous Cognitive Assessment Tool (KICA): Development of a cognitive assessment tool for older Indigenous Australians', International Psychogeriatrics, 18 (2), 269-80.

Moberg, P.J. \& Kniele, K. (2006) 'Evaluation of competency: Ethical considerations for neuropyschologists', Applied Neuropsychology, 13(2), 101-14.

Other-Gee, B., Penter, C., Ryder, L. \& Thompson, J. (2001) Needs of Indigenous people in the Guardianship and Administration system in Western Australia, Perth, Office of the Public Advocate Western Australia.

Queensland Advocacy Inc. (2004) Weaving our Magic through Connectedness, Brisbane, Queensland Advocacy Inc.

Senate Community Affairs References Committee (2006) Beyond Petrol Sniffing: Renewing Hope for Indigenous Communities, Canberra, Commonwealth of Australia.

Senior, K. (2000) Testing the ICIDH-2 with Indigenous Australians: Results of Field Work in Two Aboriginal Communities in the Northern Territory, Canberra, Australian Institute of Health and Welfare.

Setterlund, D., Tilse, C. \& Wilson, J. (1999) Substitute Decision Making and Older Australians, Trends and Issues in Crime and Criminal Justice No.139, Canberra, Australian Institute of Criminology.

Silberfeld, M., \& Checkland, D. (1999) 'Faulty judgment, expert opinion, and decision-making capacity', Theoretical Medicine and BioEthics, 20, 377-93.

Simpson, J. \& Sotiri, M. (2004) Criminal Justice and Indigenous People with Cognitive Disabilities: A Scoping Paper, Canberra, Aboriginal and Torres Strait Islander Services.

Shaddock, A.J., Spinks, A.T. \& Esbensen, A. (2000) 'Improving communication with people with an intellectual disability: The content validation of the Biala-II profile', International Journal of Disability, Development \& Education, 47 (4), 383-95.

Smith, K., Lo Guidice, D., Dwyer, A., Thomas, J., Flicker, L., Lautenschlager, N.T., Almeida, O.P., \& Atkinson, D. (2007) " "Ngana minyarti? What is this?" Development of cognitive questions for the Kimberley Indigenous Cognitive Assessment', Australasian Journal on Ageing, 26 (3), 115-9. 
Tilbury, C. (2009) 'The over-representation of indigenous children in the Australian child welfare system', Internal Journal of Social Welfare, 18, 57-64.

Tipper, D. \& Dovey, S. (1991) Bringing the Act together: The Disability Services Act and the support needs of Aboriginal people, case studies of service provision in western NSW, Sydney, Planning and Research Unit New South Wales Technical and Further Education Commission.

United Nations (2007) From Exclusion to Equality: Realizing the Rights of Persons with Disabilities Handbook for Parliamentarians on the Convention on the Rights of Persons with Disabilities and its Optional Protocol, Geneva, United Nations.

Vicary, D.A., \& Bishop, B.J. (2005) 'Western psychotherapeutic practice: Engaging Aboriginal people in culturally appropriate and respectful ways', Australian Psychologist, 40(1), 8-19. 


\section{Author Bios}

Geoff Buchanan is a PhD Candidate in Anthropology at the Centre for Aboriginal Economic Policy Research (CAEPR), The Australian National University (ANU).

Dr Hannah Bulloch is an anthropologist specialising in discourses of development. The work for this special issue was carried out while she was a Postdoctoral Fellow at the Centre for Aboriginal Economic Policy Research (CAEPR), The Australian National University (ANU). Hannah has been involved with CAEPR since early 2008, where she has worked on issues of Indigenous wellbeing and Indigenous rights to freshwater. Hannah's $\mathrm{PhD}$, which she completed in 2009, focused on Southeast Asia, exploring local notions of development on the island of Siquijor in the Philippines. Hannah is now based at the Royal Society of New Zealand and maintains a part-time Visiting Fellowship at the School of Archaeology and Anthropology at ANU.

Lesley Chenoweth is the inaugural Professor of Social Work at the School of Human Services and Social Work, and Co-Director of the Griffith Abilities Research Program, Griffith University. Her research has spanned disability issues, human services and rural communities, welfare reform, deinstitutionalisation, and program evaluation.

Jayne Clapton is the Head of the School of Human Services and Social Work, Griffith University. Her research interests are in all areas concerning people with disability, with a focus on the relationship between ethics and disability.

Natalie Clements is a senior research assistant at the School of Human Services and Social Work, Griffith University. Her experience has been in research across a range of social policy and social care issues, including people with impaired decision-making capacity, homelessness, and children in alternative care.

Dr Janet Hunt is a Fellow at the Centre for Aboriginal Economic Policy Research (CAEPR) at the Australian National University (ANU) where she is conducting research on the social benefits of Aboriginal involvement in natural resource management in New South Wales, and the work of International NGOs with Indigenous communities in Australia. She previously managed the Indigenous Community Governance Project, an Australian Research Council Linkage Project with Reconciliation Australia. She has lectured in International 
Reproduced with permission of the copyright owner. Further reproduction prohibited without permission. 